\title{
Narrativa
}

Nuova serie

$41 \mid 2019$

Narrativa italiana degli anni Duemila: cartografie e percorsi

\section{Nuovi realismi e fumetto di realtà}

\section{Giuliana Benvenuti}

\section{(2) OpenEdition \\ Journals}

\section{Edizione digitale}

URL: https://journals.openedition.org/narrativa/352

DOI: $10.4000 /$ narrativa.352

ISSN: 2804-1224

\section{Editore}

Presses universitaires de Paris Nanterre

\section{Edizione cartacea}

Data di pubblicazione: 1 décembre 2019

Paginazione: 55-64

ISBN: 978-2-84016-350-3

ISSN: $1166-3243$

\section{Notizia bibliografica digitale}

Giuliana Benvenuti, «Nuovi realismi e fumetto di realtà», Narrativa [Online], 41 | 2019, online dal 01 septembre 2021, consultato il 08 décembre 2021. URL: http://journals.openedition.org/narrativa/352 ; DOI: https://doi.org/10.4000/narrativa.352

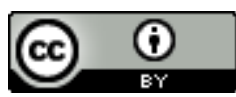

Narrativa est mise à disposition selon les termes de la Licence Creative Commons Attribution 4.0 International 


\section{Nuovi realismi e fumetto di realtà}

\section{RiAssunto}

L'articolo esplora le intersezioni tra il molto discusso "nuovo realismo" e il "fumetto di realtà", estendendo l'indagine dai territori più battuti del romanzo e del film ad altre forme espressive e tradizioni storico-artistiche. In particolare, per evidenziare come il travelogue in forma grafica sia parte della rinascita di una letteratura civile in Italia, viene proposta l'analisi degli "effetti di realtà" nei Quaderni russi di Igort.

\section{RÉSUMÉ}

Cet article explore les intersections entre le "nouveau réalisme" et la bande dessinée "réaliste", en élargissant la recherche à partir des territoires les plus explorés du roman et du film jusqu'à d'autres formes d'expression et à d'autres traditions historico-artistiques. Plus particulièrement, afin de souligner comment le travelogue graphique s'inscrit dans le cadre d'une renaissance de la littérature engagée en Italie, cet article propose l'analyse des "effets de la réalité" dans Quaderni russi d'Igort.

'ampiezza della produzione editoriale italiana, in continua crescita negli ultimi trent'anni almeno, predilige i generi di più largo consumo, come il giallo e il noir, è sempre più spesso orientata alla pubblicazione di opere spendibili sul mercato globale e rende estremamente difficile tracciare un quadro convincente delle molteplici forme assunte dal romanzo negli anni a noi più vicini. Pertanto, ci limiteremo in questa sede a segnalare un aspetto non secondario, ma ancora poco valorizzato in sede critica, ovvero la crescente presenza di iconotesti. Più in particolare, nello spazio limitato a disposizione, converrà concentrarsi su un fenomeno circoscrivibile, quello delle intersezioni tra il molto discusso "nuovo realismo" e il "fumetto di realtà", per segnalare una convergenza non scontata. Ancor più precisamente, l'intenzione di questo contributo è quella di mostrare come il travelogue in forma grafica sia 
parte della rinascita di una letteratura civile, che possiamo sommariamente definire "postmodern impegno".

Quando Maurizio Ferraris, l'estensore, è noto, del Manifesto del nuovo realismo ${ }^{2}$, e Mario De Caro mappano i "luoghi" del ritorno alla realtà ("dall'ontologia all'etica, dall'epistemologia alla semantica, dall'estetica alla filosofia della scienza"3) - da intendersi come una "filosofia globalizzata", come un sapere esteso, comprensivo tanto delle discipline umanistiche quanto di quelle sociali e naturali e capace "di rivolgersi a uno spazio pubblico" ${ }^{-}$, gli studi di settore iniziano a interrogarsi sulla (presunta) crisi del postmodernismo e sui modi del suo superamento (o della convivenza di prospettive divergenti) ${ }^{6}$.

Si ricordi, in questo senso, il numero 57 della rivista Allegoria che già nel 2008 sondava la rinnovata tensione intellettuale all'impegno, alla luce "del rapporto tra fiction e non-fiction, o meglio, del realismo negli anni della simulazione di realtà"7 e della convergenza mediale. Non a caso, la scena narrativa veniva qui esaminata congiuntamente a quella cinematografica, enfatizzando sia per la testualità verbale sia per quella visuale una spiccata vocazione reportagistica ("un affanno documentaristico e cronachistico"s) e, senza contraddizione, una ripresa della produzione di genere (soprattutto il noir, per la narrativa, e il thriller,

1. Cfr. Antonello Pier Paolo, Mussnug Florian (a cura di), Postmodern impegno. Ethics and Commitement in Contemporary Italian Culture, Oxford-Bern, Peter Lang, 2009.

2. Ferraris Maurizio, Manifesto del nuovo realismo, Roma-Bari, Laterza, 2012.

3. De Caro Mario, Ferraris Maurizio (a cura di), Bentornata realtà. Il nuovo realismo in discussione, Torino, Einaudi, 2012, p. VI.

4. Ibid., p. VIII. Il corsivo è degli autori.

5. Ibid., p. IX.

6. Ampio, e mai pacifico, il dibattito sulla processata "fine del postmoderno". Fa il punto Menegheldi Donata, "Nuovo realismo vs postmodernismo: le parole e le cose", in Contarini Silvia, De Paulis-Dalembert Maria Pia, Tosatti Ada (a cura di), Nuovi realismi: il caso italiano. Definizioni, questioni, prospettive, Massa, Transeuropa, 2016, pp. 39-56. L'attenzione sui "realismi" (nuovi e vecchi) è stata tenuta desta da numerosi interventi critici: BerTONi Federico, Realismo e letteratura: Una storia possibile, Torino, Einaudi, 2007; Guglielmi Angelo, Il romanzo e la realtà: Cronaca degli ultimi sessanta anni di narrativa italiana, Milano, Bompiani, 2010; SERKowska Hanna (a cura di), Finzione cronaca realtà: Scambi, intrecci e prospettive nella narrativa italiana contemporanea, Massa, Transeuropa, 2011; SOMIGLI Luca (a cura di), Negli archivi e per le strade: Il ritorno alla realtà nella narrativa di inizio millennio, Roma, Aracne, 2013; Palumbo Mosca Raffaello, L'invenzione del vero. Romanzi ibridi e discorso etico nell'Italia contemporanea, Roma, Gaffi, 2014; MARTín CABRERo Francisco (a cura di), Letteratura e nuovi realismi, in CoSMo. Comparative Studies in Modernism, n. 9, 2016.

7. Donnarumma Raffaele, "Nuovi realismi e persistenze postmoderne: narratori italiani di oggi”, in Allegoria, n. 57, 2008, p. 37. Più diffusamente, Donnarumma Raffaele, Ipermodernità. Dove va la narrativa contemporanea, Bologna, Il Mulino, 2014.

8. Ibid. 
per il cinema) non più soggetta a intenzioni citazionistiche o parodiche, bensì virata al ritratto socio-antropologico. "Il realismo - ricorda Raffaele Donnarumma - è un'operazione sociale: esso presuppone non solo un accordo fra il narratore e il suo pubblico, ma che il narratore rivendichi un proprio mandato". Tanto che, come nella stagione neorealistica, "appare entrato in funzione un proposito di riscoperta dell'Italia" ${ }^{10}$, chiosa Vittorio Spinazzola nel volume di Tirature (2010), che riprende il New Italian Epic del collettivo Wu Ming ${ }^{11}$ sin dal titolo, New Italian Realism. Nella stessa sede, Mauro Novelli precisa che noir e reportage, racconto di genere e cronaca, finiscono spesso per convergere nel tentativo di risuscitare un romanzo sociale a più voci dato per estinto. È "il fenomeno della cosiddetta faction, ovvero la fusione di fact e fiction" ${ }^{\prime 2}$, modulata secondo differenti strategie, perlopiù veridittive:

[...] a proliferare sono i tratti di autenticazione - su tutti la prima persona e la ricerca continua di qui-e-ora. L'accento è tutto sull'“è successo veramente", e sull'“è successo a me": su una complessiva intimazione di realtà che collega sistematicamente il testo a ciò che gli è esterno attraverso un ingombrante filtro soggettivo ${ }^{13}$.

Come procedure di rincalzo alla produzione degli effetti di verità, conviene considerare plurimi pattern, non esclusi quelli verbo-visuali. Varrà quindi la pena prestarsi a un esercizio, che a oggi ci risulta ancora intentato a ragione dello specialismo dei linguaggi in causa, provando a estendere le teorie e i concetti sopra menzionati al dispositivo intermediale del fumetto. Non si tratterà, tuttavia, di un tentativo tassonomico: a partire dal panorama contemporaneo delle narrazioni grafiche si selezionerà un oggetto specifico, il travelogue, e un autore tra $\mathrm{i}$ più noti a livello nazionale e internazionale, Igort, da analizzare nell'ottica del caso di studio, per verificare, o smentire, le ipotesi di partenza

\section{Ibid., p. 54.}

10. Spinazzola Vittorio, "La riscoperta dell'Italia", in Tirature '10. Il New Italian Realism, Milano, Il Saggiatore, 2010, p. 12.

11. Wu Ming, New Italian Epic. Letteratura, sguardo obliquo, ritorno al futuro, Torino, Einaudi, 2009.

12. Noveldi Mauro, "Il romanzo sociale senza morti ammazzati", in Tirature '10. Il New Italian Realism, cit., p. 38. Ma, lo esplicitiamo, l'obiettivo di Novelli è qui individuare un modello di romanzo sociale che racconti la metropoli o l'hinterland italiani, prescindendo dalle vicende di nera.

13. SimonetTi Gianluigi, "Gli effetti di realtà. Un bilancio della narrativa italiana di questi anni”, in Contarini Silvia, De Paulis-Dalembert Maria Pia, Tosatti Ada (a cura di), Nuovi realismi: il caso italiano. Definizioni, questioni, prospettive, cit., pp. 150-151. 
e individuare nuove aree di intervento critico ed ermeneutico. Che la scelta cada, nella ricognizione degli spazi liminari tra fiction e non-fiction, sulla letteratura di viaggio o sull'odeporica in quanto forme dell'autobiografia ${ }^{14}$, non dovrà stupire se, rifacendoci ancora a Donnarumma, consideriamo i due versanti principali della narrativa contemporanea: da un lato, infatti, "il racconto costeggia il reportage giornalistico, tra documentazione e denuncia; dall'altro, la scrittura si presenta come testimonianza veridica, recuperando i modi non dell'autofiction, ma dell'autobiografia o del racconto credibile in prima persona"15. Il travelogue (specialmente nella declinazione igortiana, come vedremo) si mostra, in questo senso, una efficace sintesi delle due modalità.

La fortuna (e l'ambiguità) dell'etichetta graphic novel presso buona parte della critica e soprattutto nel mercato editoriale ${ }^{16}$ hanno determinato un ampliamento di generi e formati, nonché dello spazio di esposizione, a disposizione del fumetto d'autore.

Se l'uso di graphic novel al posto di fumetto legittima i creatori di fumetti come autori a tutti gli effetti, il graphic novel autobiografico, attraverso un effetto di moltiplicazione e di ridondanza, li legittima anche come uomini e donne le cui vite sono degne di interesse. Non è più solo l'opera ad attirare l'attenzione, ma anche l'autore e le sue opinioni su di sé e sul mondo. [...] Qui si intende autobiografia in senso molto ampio. Palestina e altre opere di Joe Sacco sono dei reportage a fumetti in cui lo stesso Sacco, oltre a ricoprire il ruolo di narratore, si autorappresenta. Lo stesso accade [...] in Quaderni Ucraini di Igort ${ }^{17}$.

In campo letterario, il reportage associato al formato libro non è fenomeno inedito. Non dovranno pertanto stupire, pure in ambito fumettistico, le interdipendenze tra genere biografico o autobiografico e la diaristica o

14. Cfr., nel merito, BARENGHI Mario, "Ripartire dalla propria storia personale", in Tirature '10. Il New Italian Realism, cit., pp. 43-53.

15. Donnarumma Raffaele, "Nuovi realismi e persistenze postmoderne: narratori italiani di oggi", cit., p. 39.

16. Lo attesta il Rapporto dell'Associazione Italiana Editori, che ha condotto nel 2013 la prima indagine completa sul graphic novel nel mercato editoriale nostrano. Cfr. Peresson Giovanni, Vergine Elena (a cura di), Romanzi disegnati. Rapporto sul graphic novel 2013, Milano, Ediser, 2013, p. 8: "L'andamento mostra che questa produzione rappresenta circa il $10,8 \%$ della produzione di fiction. I dati [...] indicano lo spazio che il linguaggio del romanzo a fumetti inizia a occupare all'interno del più ampio linguaggio narrativo".

17. TosTi Andrea, Graphic novel. Storia e teoria del romanzo a fumetti e del rapporto fra parola e immagine, Latina, Tunué, 2016, p. 699. 
l'approfondimento giornalistico. Ma anche quella di graphic journalism, utilizzata spesso in questi casi di contaminazione, è una definizione che soggiace correntemente alle necessità di marketing: con funzione catalizzatrice, "mescola giornalismo vero e proprio con diverse forme di 'non fiction', più vicine al documentario, storico o di attualità, alla saggistica di testimonianza, alla ricostruzione storiografica o persino al memoir ${ }^{\prime \prime 18}$. La tendenza del fumetto italiano contemporaneo, non diversamente dalla produzione narrativa che ha raggiunto una vasta platea di acquirenti dalla fine degli anni Novanta a oggi, coincide sempre più col racconto (se non direttamente con la cronaca) della realtà. Espressione della controcultura americana, consolidatosi in nuovo genere dalla fine degli anni Ottanta, di "fumetto di realtà" nel contesto italiano si è cominciato a parlare con maggiore insistenza a partire dalle pubblicazioni "documentarie" di Becco Giallo Editore ${ }^{19}$ e, in particolar modo, dalle manifestazioni esplicitamente dedicate ai non-fiction comics come "Komikazen", il Festival internazionale del fumetto di realtà, organizzato annualmente (dal 2005 al 2016) a Ravenna dall'Associazione Mirada e curato da Gianluca Costantini e Elettra Stamboulis.

Questa declinazione del fumetto sembra riguadagnare una funzione critica come carattere prioritario, parzialmente contraddicendo il primo assunto di Ferraris-De Caro: "Il nuovo realismo tiene ferma l'istanza critica e decostruttiva che i movimenti antirealistici pensavano come loro esclusiva prerogativa. Anzi, i nuovi realisti vogliono recuperare gli strumenti indispensabili perché la critica sia veramente efficace: [...] la verità e la realtà" ${ }^{20}$. È, invece, proprio dal suo nucleo antirealista che la narrazione grafica dialoga col mondo circostante e ne commenta l'attualità.

È convenzione che l'innesco del processo di "ritorno alla realtà", malgrado le smentite degli scrittori interpellati a suo tempo da Donnarumma ${ }^{21}$, abbia un'origine comune in "eventi-trauma" come il crollo delle Torri Gemelle.

18. Rizzo Marco, Costantini Gianluca, Stefaneldi Matteo, "Il giornalismo a fumetti", in MicroMega, n. 3, 2018, p. 246. Ma cfr. anche FAsiolo Francesco, Italia da fumetto. Graphic journalism e narrativa disegnata nel racconto della realtà italiana di ieri e di oggi, Latina, Tunué, 2012; Gubitosa Carlo, Il giornalismo a fumetti. Raccontare il mondo col linguaggio della nona arte, Salerno, Edizioni NPE, 2018.

19. Così lo descrive il direttore editoriale Federico Zaghis: "Il nostro è un fumetto di non fiction, cronache o biografie. Dalla storia di Peppino Impastato di Marco Rizzo e Lelio Bonaccorso, il più venduto, a Che Guevara, Falcone e Borsellino. Ma anche Piazza Fontana, la strage di Bologna [...]".

20. De Caro Mario, Ferraris Maurizio (a cura di), Bentornata realtà. Il nuovo realismo in discussione, cit., p. VII.

21. Donnarumma Raffaele, Policastro Gilda (a cura di), "Ritorno alla realtà? Otto interviste a narratori italiani”, in Allegoria, n. 57, 2008, pp. 9-25. 
Più conferme (o meno reticenze), in questo senso, provengono dal versante fumettistico: pensiamo che Gipi, tra i più rappresentativi di questa generazione, arriva ad assemblare i materiali autobiografici e "sociali" della sua prima raccolta, Effetto notte (2003), proprio sull'onda emotiva del post-11 settembre ${ }^{22}$. Né è un caso che il graphic novel francese 9/11. Attentato alle Torri Gemelle (2014), misto di cronaca e ricostruzione storica e politica, sia uno dei titoli di punta della collana Historica di Mondadori Comics, che sta ospitando alcuni pregevoli esempi di "fumetto di realtà". Anche Rizzoli Lizard, in collaborazione col Corriere della Sera, nel 2013 sceglie di dedicare una collana al graphic journalism nel 2013. I curatori, Paolo Interdonato e Matteo Stefanelli, hanno selezionato venti opere tra le più celebri incentrate sull'analisi storico-geografica di situazioni socio-politiche e conflittualità etniche, dagli scenari mediorientali all'ex blocco sovietico. Qui spicca la scelta di far approdare in edicola i Quaderni ucraini di Igort: "La galassia postsovietica sembra richiedere nuove forme narrative, spesso all'insegna dell'ibridazione dei generi e dei media: abbiamo così i 'quaderni' di Igort, che combinano narrazione storica e testimonianze in prima persona" 23 .

Igort sceglie di realizzare una serie di resoconti di viaggio improntati alla volontà di far conoscere ciò che molti ignorano, con una postura vicina a quella di una firma autorevole di terza pagina, di un autore che intende raccontare al proprio pubblico colto eventi che hanno sfigurato la storia dell'ex Unione Sovietica, proiettando una lunga ombra sul presente. La quarta di copertina dei Quaderni russi presenta il libro come un reportage di viaggio che confina con l'inchiesta e impegnato nello scavo del passato, secondo le forme consuete a questo sottogenere:

Dopo l'Ucraina, la Russia sulle tracce di Anna Politkovskaja, con la stessa volontà ostinata di fare domande per capire e raccontare, Igort prosegue la sua esplorazione del mondo ex sovietico. Cos'è oggi la "democrazia travestita" dell'era di Putin, segnata dalle morti violente e misteriose di tanti oppositori? Dai giornalisti uccisi ai ceceni torturati e massacrati in una guerra dimenticata. L'autore registra storie e disegna, non con il distacco del cronista, ma con l'atteggiamento di chi si mette in cammino, ascolta le voci più deboli e vuole restare umano di fronte a fatti disumani. Un viaggio indimenticabile

22. Cfr. Interdonato Paolo, "Il fumetto è reale: cronache e autobiografismo nel graphic novel", in Tirature '10. Il New Italian Realism, cit., p. 58.

23. Sullam Sara, "Graphic journalism e romanzi storici spagnoli”, in Tirature '14. Videogiochi e altri racconti, Milano, Il Saggiatore, 2014, ed. digitale. 
nella memoria e nell'anima russa. Un appello a non voltare la testa dall'altra parte. Un libro che ci interroga a ogni pagina ${ }^{24}$.

Richiamando un lavoro di inchiesta più ampio, che vede nei Quaderni russi la propria prosecuzione, la quarta presenta l'autore come un intellettuale impegnato a far conoscere aspetti oscuri della storia che non devono essere dimenticati, che interpellano direttamente l'umanità dei lettori, mettendola alla prova, così come è avvenuto all'autore e al suo modello, Politkovskaja, centro della narrazione. Il sottotitolo, come quello dei Quaderni ucraini ${ }^{25}$, iscrive il volume all'interno del genere dei "reportages disegnati", confermando l'intenzione di presentarlo quale parte di una tradizione consolidata.

Dopo un iniziale scetticismo nei confronti del fumetto politico (almeno nella sua accezione mainstream $)^{26}$, Igort si rivolge al travelogue come a un genere congeniale alla propria vocazione narrativa, dando prove plurime e diverse ${ }^{27}$. Nella trilogia, composta da Quaderni ucraini, Quaderni russi e Pagine nomadi emerge la mescidanza tra racconto di viaggio, ricostruzione memoriale e vocazione alla

24. Igort, Quaderni russi. Sulle tracce di Anna Politkovskaja. Un reportage disegnato, Milano, Mondadori, 2011 (poi Bologna, Coconino Press, 2014).

25. Igort, Quaderni ucraini. Le radici di un conflitto. Un reportage disegnato, Milano, Mondadori, 2010 (poi Bologna, Coconino, 2014).

26. Cfr. IGort, "Le facce del fumetto", 16 gennaio 2006, http://igort.blogspot. com/2006/01/le-facce-del-fumetto.html: "Il fumetto politico o con agganci sulla realtà sembra essere un argomento che sta a cuore a molti. Persone diverse. Anche legate ad altri media, non lettori abituali, vorrebbero avere un moltiplicarsi di reportage a fumetti, per esempio; o un tipo di 'osservazione sociale'. [...] a me il fatto che la trasmissione di Radio 3, Fahrenheit, debba sempre legare un romanzo alla cronaca del telegiornale mi lascia interdetto. Lolita lo dobbiamo leggere alla luce di una retata antipedofila? Pastorale americana legato all'infiltrazione terrorista sul territorio americano? Ma questo non toglie che mi interessa leggere Joe Sacco e vedere come filtra il suo sguardo personale con le tinte più oggettive da giornalista". Igort affida al blog (e dunque, al filo diretto col lettore) la decodifica del fumetto non-fiction: quello in cui il racconto dei fatti e la prospettiva autoriale si compenetrano, vicendevolmente rafforzandosi.

27. Si pensi, ad esempio, ai Quaderni giapponesi. Un viaggio nell'Impero dei segni, Bologna, Coconino Press, 2015 (il secondo volume Quaderni giapponesi. Il vagabondo del manga è uscito per i tipi di Oblomov Edizioni nel 2017) che fin dal titolo rinvia a Roland Barthes, confermando l'intenzione colta di questa come delle altre opere, dove troviamo una ibridazione tra diario di viaggio e saggio disegnato che pone in atto rimarchevoli processi di transculturazione. La passione di Igort per la cultura giapponese ha dato forma a un racconto saggistico-autobiografico che potremmo collocare nella tradizione della narrativa di viaggio di terza pagina, non fosse che il disegno consente di mostrare una infinita gamma di risonanze provenienti dall'arte giapponese, che il solo mezzo scritto difficilmente riuscirebbe a evocare. 
denuncia, resa esplicita anche dal sottotitolo del terzo volume, Pagine nomadi. Pagine non ufficiali dall'ex Unione Sovietica ${ }^{28}$, che riporta appunti di viaggio, immagini e testi delle interventiste effettuate dall'autore e utilizzate ampiamente nei primi due volumi. Il metodo e le forme del racconto concorrono a fare di questi reportage un esempio notevole di mescolanza tra travelogue, inchiesta giornalistica, ricostruzione memoriale, benché il loro autore ami definirsi (come accade anche nel suo blog, dove non pubblica tavole e non parla solamente di fumetto) uno storyteller:

Non mi considero un cronista, né un autobiografo, ma semplicemente un narratore. Ecco tutto. E la cultura russa e ucraina l'ho visitata come si visitano le proprie stanze interiori, con un pudore misto a desiderio di conoscere, magari, in ultima istanza di capire ${ }^{29}$.

Nei Quaderni russi Igort esplicita il suo legame personale con la figura di Anna Politkovskaja. In virtù di questo legame, di questa affinità, l'autore sente la necessità di raccontare gli aspetti e gli eventi della vita della giornalista che hanno portato alla sua uccisione, intrecciandoli con la narrazione delle violenze delle quali Anna Politkovskaja era stata testimone, sulle quali indagava per farle conoscere. La sua vicenda è per Igort esemplare di un modo di essere nel mondo che resiste umanamente prima ancora che politicamente, alla violenza e al terrore. Politkovskaja rappresenta un modello umano e professionale, le due cose insieme e inscindibili, dal momento che la giornalista ha incarnato un eroismo non retorico, che con tutta evidenza è la maniera di svolgere la professione che Tuveri, in arte Igort, ci indica come esemplare. La resistenza personale, la necessità di non abdicare alla propria umanità, intesa come volontà di denunciare, certo, ma prima ancora di comprendere la sofferenza delle vittime, e anche, per quanto aberrante, l'umanità deviata e distorta dei carnefici, sono al centro della ricostruzione operata da Igort della figura della giornalista, che interpella il lettore, e ancor prima l'autore. Politkovskaja è un esempio di coraggio e di umanità. Oltre a questo, ciò che occorre rilevare è che le sue pagine offrono un modello di scrittura capace di coinvolgere empaticamente il lettore, che Igort segue nei propri resoconti. Per questo, il reportage di viaggio, in parte anomalo, dal momento che l'autore ripercorre la storia di un personaggio significativo attraverso un

28. Igort, Pagine nomadi. Pagine non ufficiali dall'ex Unione Sovietica, Bologna, Coconino Press, 2012.

29. IGORT, "I quaderni mistici di Igort", Lo Spazio bianco, 19 aprile 2012, https:// www.lospaziobianco.it/quaderni-mistici-igort/ 
pellegrinaggio nei luoghi che ha abitato e le interviste o i dialoghi con chi lo ha conosciuto, non si limita a costruire una sorta di auto-mito-biografia della giornalista, bensì riporta le impressioni del viaggiatore e i tormenti della scrittura, dando conto al lettore, con inserti metanarrativi, della difficoltà (tanto di Politkovskaja, quanto di Igort) di raccontare storie di tortura, mutilazione, umiliazione, dal punto di vista di chi ha subito le violenze della storia.

Mi interessa una scrittura che si interroghi - afferma Igort -, una scrittura morale se vogliamo. E mostrare anche che l'atroce fa, in qualche misura, parte di questo. Ecco. Sono piccoli, minuscoli, passi, che compio in privato, cercando di calpestare sentieri che altri senz'altro più nobili di me, in altre discipline hanno percorso ${ }^{30}$.

La narrazione è condotta attraverso un uso parco dei balloon, ampio, invece, delle didascalie e del testo scorporato. Sulla scorta dell'analisi dei Quaderni ucraini condotta da Valerio Monti ${ }^{31}$, possiamo notare che nei Quaderni russi il testo scorporato è un elemento fondamentale delle parti di approfondimento storico e in generale è molto presente, al pari delle didascalie, mentre sono rari i momenti della progressione narrativa lasciati solamente alle immagini e al contenuto dei balloon. La componente iconografica, come afferma Monti, contribuisce al ritmo narrativo, ma soprattutto al coinvolgimento emotivo: l'uso dei colori non è solamente o principalmente mimetico, bensì emotivamente connotato, specie là dove vengono mostrate le violenze su corpi inermi, e genera un effetto di senso, seguendo "il valore che il contesto culturale attribuisce tradizionalmente a una determinata tinta"32. Anche le scene più cruente risultano, in virtù del carattere evocativo delle immagini e del testo che le accompagna, lontane dal rischio della esibizione gratuita della violenza, riuscendo a creare empatia verso la sofferenza e indignazione, in coerenza con le intenzioni del racconto.

In breve, Igort, che giunge al reportage disegnato dopo una carriera che lo ha visto protagonista della sperimentazione del gruppo Valvoline, tra i primi in Italia a perseguire una poetica vicina al modello del romanzo disegnato, accreditato fumettista le cui opere sono tradotte in più lingue, fondatore di molte riviste, vincitore di un numero rimarchevole di premi, fondatore di una impresa editoriale di rilievo come la Coconino Press e ora di Oblomov Edizioni,

\section{Ibid.}

31. Monti Valerio, “I 'Quaderni ucraini' di Igort. Analisi linguistica di un reportage a fumetti”, in Italiano LinguaDue, n. 1, 2013, pp. 243-294.

32. Ibid., p. 267. 
si presenta al lettore quale autore affermato, consapevole della propria competenza nell'uso degli strumenti espressivi propri della narrazione lunga, autoconclusa, autoriale del fumetto e propone un esempio di reportage grafico che interseca narrazione storica, memoriale e autobiografica, per il quale possiamo riprendere le conclusioni alle quali giunge Monti:

[...] l'autore non è vincolato dai parametri impliciti presenti nel fumetto seriale: il pubblico a cui si rivolge non ha una specificità legata a un particolare genere di fumetto. Il lettore è consapevole del carattere di originalità e di unicità dei Quaderni ucraini, che si presenta, ancor prima che come reportage a fumetti, come un graphic novel d'autore; la lingua è dunque libera dai canoni vigenti nel fumetto mainstream, in cui l'aspetto linguistico deve soddisfare gli orizzonti d'attesa del pubblico. [...] Il risultato finale è una varietà di lingua non trascurata, puntuale ed allo stesso tempo facilmente fruibile da un pubblico ampio e trasversale, nata dalla capacità dell'autore di bilanciare le diverse componenti sia linguistiche che iconografiche che costituiscono la sua opera ${ }^{33}$.

Una scrittura colta, soprattutto sul piano iconografico, è tra gli "effetti linguistici di realtà" 34 con cui Igort sigla la sua adesione al "nuovo realismo", a dimostrazione, anche se attraverso un singolo caso di studio, di quanto sia opportuno, o forse necessario - al fine di cartografare le modalità narrative che la contemporaneità richiede per essere raccontata - estendere l'indagine dai territori più battuti del "nuovo realismo" romanzesco e filmico ad altre forme espressive e tradizioni storico-artistiche.

Giuliana Benvenuti Università di Bologna

33. Ibid., p. 289.

34. SimONETTI Gianluigi, "Gli effetti di realtà. Un bilancio della narrativa italiana di questi anni”, cit., pp. 159-160. 